\title{
THEORY OF FLARES
}




\title{
Physical Processes During Impulsive Solar and Stellar Flares
}

\author{
Maria Katsova ${ }^{1}$, Moissei Livshits ${ }^{2}$ \\ 1 Sternberg State Astronomical Institute, Moscow State University, \\ 119899 Moscow, Russia \\ 2 Institute of Terrestrial Magnetism, Ionosphere and Radio Wave Propagation, \\ Russian Academy of Sciences, 142092 Troitsk, Moscow Region, Russia
}

\section{Introduction}

Investigations of impulsive flares on both the Sun and red dwarf stars during more than 30 years allow us to arrive at quite definite conclusions. Here we will consider impulsive events; on the Sun the impulsive phase of a flare is observed as a hard X-ray burst with the emission of photons with energies $E>30 \mathrm{keV}$ up to the $\gamma$-ray range. At the same time microwave radio bursts, and sometimes UV and optical continuum bursts are registered. Typical durations of these processes are $\sim 1-3 \mathrm{~min}$. In this time interval other kinds of flare emission like soft $\mathrm{X}$-ray (2-10 keV) emission, meter radio bursts and Balmer line emission begin to rise, but their maxima occur later on, in the gradual (thermal) phase of the flare.

Impulsive stellar flares are often observed as a significant increase in optical continuum, especially in the U-band, of similar duration (1-3 min), and this time interval is, like in the solar case, the rise phase of the soft $\mathrm{X}$-ray emission.

Modern observations demonstrate that both the impulsive phase of a flare or an impulsive flare develops in low-lying loops. Earlier only indirect evidence existed in optical and radio data. Recently, however, the heights of the hard Xray sources in impulsive solar events were determined directly from YOHKOH's HXT (Kosugi 1994, Masuda 1994) (Fig. 1a). Statistically, the height of the hard $\mathrm{X}$-ray source in the $14-23 \mathrm{keV}$ range is $9700 \pm 2000 \mathrm{~km}$ above the photosphere, and this height reduces to $6500 \mathrm{~km}$ in the $53-93 \mathrm{keV}$ range. Besides two hard $\mathrm{X}$-ray sources in the loop footpoints, a third hard X-ray source exists at the top of the loop at least in some cases. The authors of this experiment suppose that the appearance of this loop-top source is due to reconnection in the impulsive phase. Note that the reconnection begins close to the apex of the loop, when this loop is filled by hot plasma that evaporated from both footpoints.

Thus, the impulsive phase of large flares takes place at low chromospheric heights $(<10000 \mathrm{~km}$ ), often close to a spot (Fig. 1b). In the process electrons are accelerated and impact on the chromosphere adjacent to the footpoints of the low-lying loop. The response of an atmosphere to the action by primary accelerated particles and by thermal and radiation fluxes is referred to as secondary processes in a flare and will be discussed separately below. The impulsive phase 

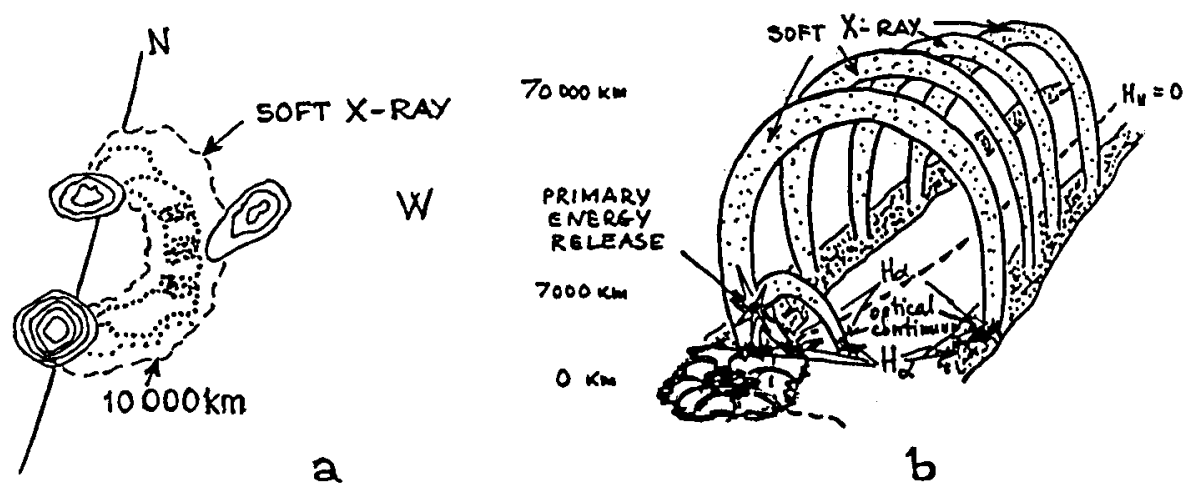

Fig. 1. (a) The brightness distribution in the $14-23 \mathrm{keV}$ and the soft $\mathrm{X}$-ray ranges according to Kosugi (1994) and Masuda (1994); (b) The system of flare loops: low-lying impulsive source and high coronal loop.

lasts 1-3min and later accelerated particles and hot plasma go into the higher coronal loops, and the main soft X-ray source is formed (Katsova \& Livshits 1991).

During evolution of magnetic fields or the motion of one spot relative to others, or when new spots emerge, energy is stored gradually in the non-potential part of the magnetic field. In large flares magnetic tensions are relaxed and the magnetic field lines return toward a potential configuration as the excess energy is released. The process can be repeated up to a few times leading to series of homologous flares while active regions are on the solar disk.

In some cases the motions of magnetic hills or the emergence of magnetic flux can be observed, but as a rule it is difficult to detect these effects directly. It is clear, however, that the impulsive phase begins close to spots, often in their penumbrae, near to photospheric bridges or to a neutral line of longitudinal magnetic field. Zirin \& Wang (1993a) emphasize that big flares occur in regions with very strong transverse magnetic field $(1000-2000 \mathrm{G}$, e.g. on 4 June 1991 $-4000 \mathrm{G}$ ). The motion of photospheric footpoints does form extremely narrow elongated channels of intense horizontal field (Zirin \& Wang 1993b). In such regions the strongest impulsive solar flares take place, like the events of June 1991.

Magnetic fields of an unipolar spot are more squeezed to the photosphere in the direction where they confine the points of the umbrae and bright points of the chromospheric network, i.e. in these azimuths the "canopy effect" is observed (Zhang 1994). Magnetic force lines of other azimuths of an unipolar spot extend up to large heights, like in the case of the field of a vertical dipole. It is quite possible that flare activity is enhanced in those places where this "canopy" effect is present. 
At the onset of an impulsive flare particles, particularly electrons, are accelerated. This primary acceleration takes place in a very small volume. The total energy of fast particles exceeds the energy of the magnetic field in this volume, therefore the question remains (see also van den Oord 1993): how does the globally stored energy dissipate locally?

Energy storage can occur in large-scale current flows. The current intensities and changing of electric conductivity and other parameters provide the power, and the duration of the acceleration can be estimated using an equivalent circuit model (Spicer et al. 1986, Stepanov \& Zaitsev 1992, Melrose, 1994). However, it is required that this theory be able to describe such a process in the real solar atmosphere, not only in ideal circuits.

The details of the process of primary energy release are still unknown. X-ray data provide evidence for extremely fast and very effective particle acceleration. From a theoretical point of view a trigger mechanism is required and can be connected with an instability of a ring current (like the pinch-effect); it is necessary to provide such a mechanism of particle acceleration operating for a few seconds at least.

At present, it is becoming clear that both in the Earth's magnetosphere and in the magnetospheres of solar active regions several mechanisms of particle acceleration should be taken into account. Strong non-linear plasma effects, like solitons, can be significant.

Outstanding problems are: to give a theory of the evolution of particle beams in coronal magnetic tubes, to explain the radio spectra in the entire range from meter to millimeter, and to explain the flat spectra of microwave type-IV radio emission. If we could know how the energetics and the angular distribution of particles change in a magnetic trap, we could compute the gyrosynchrotron radiation of accelerated particles to explain brightness temperatures as high as $3 \cdot 10^{9} \mathrm{~K}$.

\section{Accelerated particles in impulsive solar flares}

There are three main types of observations from which we can derive the properties of accelerated particles: hard X-ray and $\gamma$-ray bursts and direct registration of accelerated particles in interplanetary space and at the Earth. The differential spectrum of the X-ray emission with $E>E_{1}=15 \mathrm{keV}$ can be described as

$$
I(h \nu)=I_{0}(h \nu)^{-\delta} \quad \text { photons } / \mathrm{cm}^{2} \mathrm{~s} \mathrm{keV}
$$

where the values of the fluxes $I, I_{0}$ and the index $\delta$ of the power law depend on the time.

The solution of the Abel integral equation in the Born-approximation for the differential cross-section of bremsstrahlung radiation allows us to determine the number of electrons in the emission source:

$$
N_{\mathrm{e}}(E, t) \approx 10^{42} \frac{I_{o}}{n} \frac{(\delta-1) \Gamma(\delta-1 / 2)}{\Gamma(\delta-1) Z^{2}} E^{-(\delta-1 / 2)} \text { electrons } / \mathrm{keV}
$$


where $n$ is mean ion density in the emission source, $Z^{2} \approx 1.8$ for cosmic plasmas and $\Gamma(x)$ is the $\Gamma$-function.

From the solution of the equation of continuity for accelerated particles the particle spectrum in the region of the acceleration is

$$
F_{o}(E, t) \approx 5 \cdot 10^{-9} \delta n E^{-3 / 2} N_{e}(E, t) \text { electrons } / \mathrm{s} \mathrm{keV}
$$

These equations are based on Brown's (1971) pioneering paper and some later considerations (Syrovatskij \& Shmeleva 1972, Korchak 1976).

Using Eqs. 1-3 it is possible to derive the flux of electrons with $E>15$ $\mathrm{keV}$ as $10^{36}$ electrons/s for a typical solar hard X-ray burst; the total number of electrons accelerated in a large impulsive solar flare is $10^{38}$. From here the electron energy input to the low-lying chromosphere is $1-3 \cdot 10^{11} \mathrm{erg} / \mathrm{cm}^{2} \mathrm{~s}$, for bright points of area $\sim 10^{17} \mathrm{~cm}^{2}$.

During some flares significant numbers of electrons are accelerated to $10 \mathrm{MeV}$, as observed with the gamma-spectrometers on $S M M$ and YOHKOH. Besides bremsstrahlung radiation of accelerated electrons, a number of nuclear lines are detected with the same $S M M$ and YOHKOH equipment. To explain the appearance of these lines it is necessary to accept that $10^{33}$ protons are accelerated up to energies $\geq 30 \mathrm{MeV}$ (Ramaty 1986). These same observations give evidence, apparently, for the existence of a cut-off in the proton spectrum beyond $700 \mathrm{MeV}$ (Alexander et al. 1994).

Analysis of solar cosmic ray increase supports both the above conclusions concerning the proton spectrum. So, large number of protons, up to $2 \cdot 10^{33}$ with $E>30 \mathrm{MeV}$ were accelerated in the solar flare on 24 May 1990 and in the impulsive phase of the flare on 15 June 1991 (Belov \& Livshits 1995, Belov et al. 1994). These data contain the only hint of the presence of a cut-off of the proton spectrum at $700 \mathrm{MeV}$.

The proton spectrum in the flare of 29 September 1989 which occurred behind the solar limb does extend up to very high energies. Here the impulsive phase could not be observed directly and the registered accelerated particles originated from the very high corona. For other flares with an expressed impulsive phase, the proton spectrum beyond $700 \mathrm{MeV}$ turns out to be sharper (Fig. 2 from Belov \& Eroshenko 1995). At the moment this problem is under more detailed investigation.

However, it is possible to propose the following preliminary conclusion for the most powerful solar flares. The length of the current sheet even in the most powerful impulsive solar flares is not sufficient to provide acceleration of particles up to energies of a few $\mathrm{GeV}$, while in some events, after the pulse, the current sheet can reach a solar radius in length. Then the particles can be accelerated up to energies of $10^{10} \mathrm{eV}$.

Modern information on accelerated particles is sufficient for a correct estimation of the production of light elements during flares. 


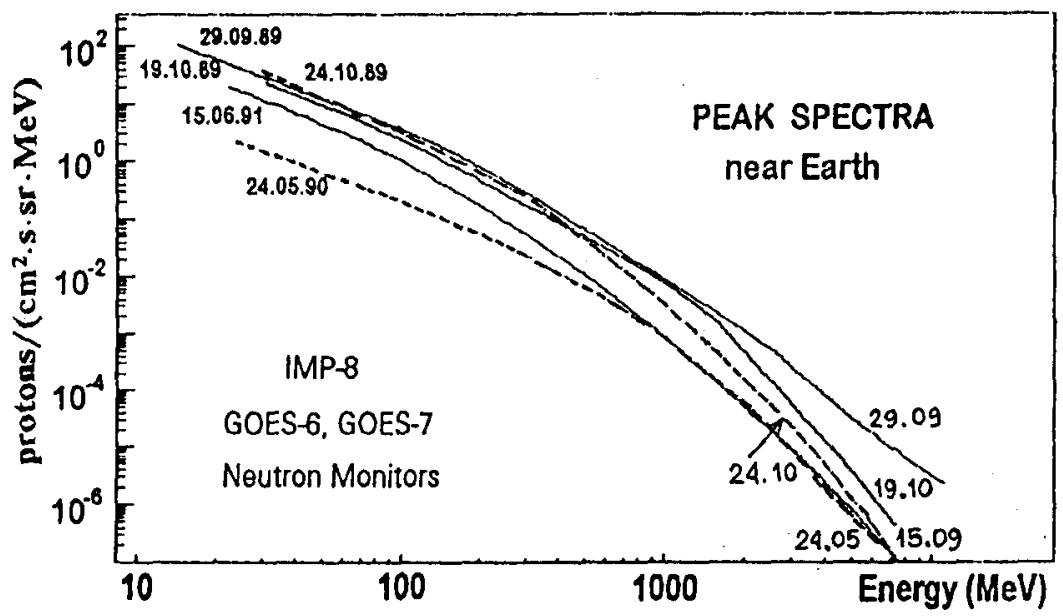

Fig. 2. The proton spectrum observed near the Earth at the maximum of the largest enhancement of solar cosmic rays during solar cycle XXII.

\section{The response of solar and stellar chromosphere to impulsive action}

The first discussion of the analogy between solar and stellar flares was that of Gershberg \& Pikel'ner (1972). Attention was drawn to the problem of the primary energy release which on both the Sun and the stars is the result of magnetic field evolution. The paper by Kostyuk \& Pikel'ner (1975) was fundamental in the serious study of secondary processes in solar flares. This question was further investigated in detail by Fisher (1986, and references therein), and general results of gas-dynamical simulations were reproduced by ten groups (Kopp et al. 1989). As a source of heating one more often considers accelerated electrons, although both protons and EUV or soft X-rays are able to make significant contributions in some cases.

The different influences of particle beams and the heat fluxes from action of $\mathrm{X}$-rays need to be emphasized: the accelerated particles and heat fluxes propagate along magnetic field lines and lead to the appearance of soft $\mathrm{X}$-ray and optical emission in separate bright points (close to the loop footpoints). Flaring coronal X-ray emission illuminates all the low-lying area and results in a diffusion "halo" source of the UV and optical radiation.

The process of chromospheric response to impulsive heating distributed over column mass, i.e. the process of explosive evaporation, develops on the Sun in the following way. In the first tenth of a second the upper chromosphere is heated suddenly without noticeable change of total density, because motions are still absent. Two disturbances propagate, upwards and downwards, from the resulting zone of high pressure. One second after the beginning of heating the quasi-stationary plasma flow moves downwards and a shock wave forms. The gas behind this radiative shock front is compressed by about a factor of 100 and a ge- 
ometrically thin emission source, with $T=10^{4} \mathrm{~K}$ and high density, forms. This source is called "the low-temperature condensation". This disturbance propagates downwards. Hot plasma evaporates from this region, and becomes the source of soft X-ray emission.

The effects predicted by the theory of gas-dynamic processes are observed directly in impulsive solar flares. First, the expected outflow of hot gas into the corona during the hard phase was detected with SMM (Antonucci et al. 1982). Second, the properties of the $\mathrm{H} \alpha$ emission in bright points in the impulsive phase agree well with those calculated in models (Bruevich 1995).

The first calculations of explosive evaporation in the atmosphere of red dwarfs were carried out by Livshits et al. (1981) and Katsova et al. (1981). Their main conclusion was that the low-temperature condensation formed in this process during large flares should emit in optical continuum. This feature of the solution is due to the higher density of red dwarf atmospheres as compared to the solar case.

Recently we carried out a new modeling of this gas-dynamical process for flares on AD Leo, using modern numerical methods (Boiko \& Livshits 1995, Katsova et al. 1995). The system of equations of gas-dynamics for $2 T$ - and onefluid plasma includes heating flux, radiative energy losses, thermal conduction flux and the function of the energy change between the electron and ion component of plasma. Previous experience showed that the usual Lagrangian coordinates should not be used, and we adopted the motions of the sites of the numerical grid in order to compute exactly the shock wave and the thermal conduction fronts. This numerical method of solution is similar to the mixed Euler-Lagrangian approach.

As an initial model we used the Hawley-Fisher model for the chromosphere and transition region and then added coronal layers. We supposed that the chromosphere is heated by an electron beam of energy of $3 \cdot 10^{11} \mathrm{erg} / \mathrm{cm}^{2} \mathrm{~s}$ and the temporal profile of the heating flux has a rectangular form. The computations have been carried out for one elementary burst lasting $10 \mathrm{sec}$. Real stellar flares can be represented as a set of such bursts. The propagation of the lowtemperature condensation downwards through the red dwarf chromosphere is shown in Fig. 3.

This gas-dynamical computation allows us to explain many features of simultaneous observations of flares in different spectral ranges. Optical continuum emission of the low-temperature condensation has a thermal origin. This is supported at the present by the UV-continuum observations. The area of optical flares is about of $(1-5) \cdot 10^{18} \mathrm{~cm}^{2}$. The temperature of the source of optical emission is in the range $8000-15000 \mathrm{~K}$. It is worth noting that the temperature of the condensation as a whole should be determined by taking into account the absorption therein of the soft X-ray emission. This effect was analyzed by Hawley \& Fisher (1992).

Some features such as, e.g., the evolution of the Balmer decrements and the appearance of broad components of the line profiles, can be explained in the framework of this model. Part of the line emission is caused by radiation of the 


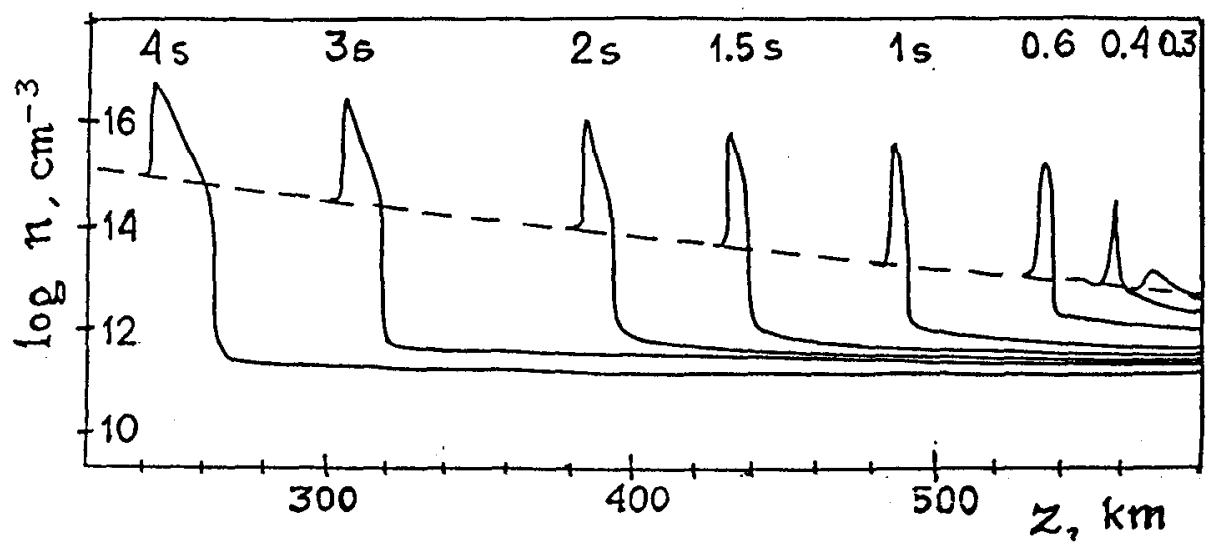

Fig. 3. The density distribution versus the height in the stellar chromosphere. The null height is photospheric level where $\tau(5000 \AA)=1$. The time in seconds after the beginning of heating is indicated. (Katsova et al. 1995).

low-temperature gas above the flare region.

UV (1000-2000 $\AA$ ) emission should accompany the beginning of each elementary process of explosive evaporation (Katsova \& Livshits 1989). Furthermore, we found one new feature of our solution: regions with high pressure arise from time to time close to the thermal conduction front due to specific conditions of the heat propagation. The motions restore the previous flow. However, the process of isruption of the hot-cool gas boundary should be accompanied by additional UV emission. Thus, the EUV light curve should show a set of spikes of short duration.

It is also worth noting that the evolution of the source of soft X-ray emission can be explained as well.

Intergrated over the duration of a flare, energies emitted in the X-ray, UV and optical spectral range should be close to each other. This theoretical conclusion is in agreement with observations.

\section{Concluding remarks}

We are happy to understand something about the nature of solar and stellar flares, for instance, the thermal origin of the optical continuum emission. However, a number of questions are far from a final solution. Among other things:

- the real relationship between magnetic field motions in or above spots and the impulsive flare onset,

- high energy processes and the interpretation of radio emission of stellar flares,

- verification of the presence of short UV spikes in solar and stellar flares. 


\section{References}

Alexander D., Dunphy P.P., MacKinnon A.L., 1994, Solar Phys. 151, 147

Antonucci E., Gabriel A., Acton L., et al., 1982, Solar Phys. 78, 107

Belov A.V., Eroshenko E.A., 1995, Nuclear tracks and radiation measurements (in press)

Belov A.V., Eroshenko E.A., Livshits M.A., 1994, Proc. 8th Int. Symp. on SolarTerrestrial Physics, Sendai, Japan Pt. 1, 26

Belov A.V., Livshits M.A., 1995, Sov. Astron. Lett. 21, 1

Boiko A.Ya., Livshits M.A., 1995, Astron. Zh. 72, (in press)

Brown J., 1971, Solar Phys. 18, 489

Bruevich E.A., 1995, Astron. Zh. 72, No. 1

Fisher G.H., 1986, Radiation Hydrodynamics in Stars and Compact Objects, Lect. Notes in Physics, 255, 53

Gershberg R.E., Pikel'ner S.B., 1972, Comments Ap. Space Phys. 4, 113

Hawley S.L., Fisher G.H., 1992, ApJS, 78, 565

Katsova M.M., Livshits M.A., 1989, Sov. Astron. 33(2), 155

Katsova M.M., Livshits M.A., 1991, Sov. Astron. 35(1), 65

atsova, M.M., Boiko A.Ya., Livshits M.A., 1995, (in prep.)

Katsova, M.M., Kosovichev A.G., Livshits M.A., 1981, Astrofiz. 17, 285

Kopp R.A., Fisher G.H., MacNice P., et al., 1989, Energetic Phenomena on the Sun, Kundu M.R., Woodgate B., Schmahl E.J. (eds.), Kluwer, Dordrecht, p. 601

Korchak A.A., 1976, Astron. Zh. 53, 370

Kostyuk N.D., Pikel'ner S.B., 1975, Sov. Astron. 18, 590

Kosugi T., 1994, New Look at the Sun with Emphasis on Advanced Observations of Coronal Dynamics and Flares. Proc. of Kofu Symp. NRA Rep. No. 360, Enome S. \& Hirayama T. (eds.), p. 11

Livshits M.A., Badalyan O.G., Kosovichev A.G., Katsova M.M., 1981, Solar Phys. 73, 269

Masuda S., 1994, PhD Thesis. Univ. of Tokyo

Melrose D.B., 1994, New Look at the Sun with Emphasis on Advenced Observations of Coronal Dynamics and Flares, Proc. of Kofu Symp. NRA Rep. No. 360 eds. Enome S. \& Hirayama T. (eds.), p. 235

van den Oord G.H.J., 1993, Advances Space Res. 13, No. 9, 143

Ramaty R., 1986, Physics of the Sun, Sturrock, P. et al. (eds.) Reidel, Dordrecht, Vol. 2,291

Spicer D.S., Mariska J.T., Boris J.P., 1986, Physics of the Sun, Sturrock, P. et al. (eds.) Reidel, Dordrecht, Vol. 2, 181

Stepanov A.V., Zaitsev V.V., 1992, Solar Phys. 139, 343

Syrovatskij S.I., Shmeleva O.P., 1972, Astron. Zh. 49, 334

Zirin H., Wang H., 1993a, Solar Phys. 144, 137

Zirin H., Wang H., 1993b, Nature 363, 426

Zhang H., 1994, Solar Phys. 154, 207 